\title{
Numerical Modeling of a High Repetition Rate Fiber Laser, Mode - Locked by External Optical Modulation of a Semiconductor Optical Amplifier
}

\author{
K. Zoiros, T. Stathopoulos, K. Vlachos, A. Hatziefremidis, \\ T. Houbavlis, T. Papakyriakopoulos and H. Avramopoulos \\ National Technical University of Athens Department of Electrical and Computer \\ Engineering, Photonics Communications Research Laboratory, 9 Iroon Polytechniou \\ Street, 157 73, Zografou, Athens, Greece, Tel : +30 1772 2076, Fax : +30 1 7722077, \\ Email : hav@cc.ece.ntua.gr
}

Key words: Active mode - locking; High repetition rate ring laser; Semiconductor optical amplifier; External optical modulation

\begin{abstract}
A theoretical study of a high repetition rate laser source based on a novel mode locking technique is presented. This technique relies on the fast saturation and recovery of a semiconductor optical amplifier induced by an external optical pulse and has been used to obtain 4.3 ps pulses at $20 \mathrm{GHz}$. A numerical model of the fiber ring laser has been developed describing the mode - locking process in the laser oscillator and providing solutions for the steady - state mode - locked pulse profile. The critical parameters of the system are defined and analyzed and their impact on the formation of the mode - locked pulses is examined. The comparison between the theoretical results and the experimental data reveals very good agreement and has allowed the optimization of the performance of the system in terms of these parameters.
\end{abstract}

\section{INTRODUCTION}

Ultra-high speed multi-access networks are evolving rapidly and are being developed to satisfy the increasing bandwidth demand due to the massive 
use of Internet and multimedia. Optical signal sources capable of generating stable ultra - short pulse trains at high repetition rates and with a tuning range as wide as possible are key elements for these novel photonic networks that may combine wavelength division multiplexing (WDM) and optical time domain multiplexing (OTDM) transmission techniques [1]. Short pulse ultrafast lasers are also necessary for the performance of ultra high speed, all - optical logic experiments and the demonstration of all optical Boolean functions such as AND and XOR [2], [3]. Active mode locking is one of the main methods for the generation of ultra - short transform - limited optical pulses which is based on the direct modulation of the optical field during each cavity round - trip. At $1.5 \mu \mathrm{m}$, several actively mode - locked fiber lasers employing Er doped fiber as the gain medium and producing transform - limited picosecond pulses at multi - $\mathrm{GHz}$ rates have been demonstrated [4 - 9]. The majority of these systems uses loss modulation by lithium niobate electro - optic modulators due to their large electro - optic coefficient and their compact construction on low loss titanium - indiffused waveguides. Unfortunately, these modulators are highly sensitive to the polarization state of the input optical field. For this reason, laser sources using lithium niobate modulators have to be build from polarization preserving fiber pigtailed components [6], [7] or else suffer from mode - locking loss which limits their operational usability. Similarly, the use of lightly or moderately doped Er fiber results in long cavities which make fiber lasers sensitive to small environmental perturbations, such as thermal fluctuations and acoustic vibration. Although several active stabilization techniques have been developed to counter the instability of long cavity fiber lasers, these increase the complexity of the system [8], [9]. In order to solve these problems, semiconductor optical amplifiers (SOAs) have been deployed in mode - locked laser sources for the generation of short optical pulses at high repetition rates. The use of an SOA inside the laser cavity is particularly attractive since it can provide simultaneously gain over a broad spectral range and modulation due to its fast gain dynamics. Actively mode - locked laser sources with an intracavity SOA have been demonstrated by several research groups [10 - 15] to generate short optical pulses at various repetition rates. In these configurations the SOA provides either the necessary gain exceeding the ring laser cavity loss in combination with an intracavity electroabsorption [10] or electrooptic modulator [11] or both gain and electrically controlled gain modulation [12 - 15].

This paper presents theoretical results obtained from an actively mode locked fiber ring laser source that uses a single SOA to provide both gain and gain modulation via cross gain saturation from an external optical pulse train. The novel configuration of the fiber ring laser source results in a stable oscillator since the use of a single active element to provide both gain and modulation renders the cavity short. Furthermore, the cavity is nearly polarization insensitive as there is no lithium niobate modulator and the SOA 
is operated under heavy saturation providing nearly polarization independent gain. The source is very simple to build from commercially available components and has formed a technology platform from which four subsystems of exceptional good operating performance have been demonstrated. These subsystems are crucial for the implementation of high speed telecommunication transmission and network systems that are based on WDM and OTDM techniques. More specifically, these subsystems include (a) a 4.3 ps stable $20 \mathrm{GHz}$ fiber ring laser source with $16 \mathrm{~nm}$ tuning range [16], (b) a $10 \times 30 \mathrm{GHz}$ source which generates 10 synchronized wavelength channels, each mode-locked at $30 \mathrm{GHz}$, producing nearly transform limited 7 ps pulses with less than $5 \%$ power variation across them [17], (c) an optical clock repetition rate multiplier circuit which has achieved a factor of 6 frequency multiplication and up to $34.68 \mathrm{GHz}$ [18] and (d) an optical clock recovery and division circuit capable to recover clock from a data pattern up to $20 \mathrm{~Gb} / \mathrm{s}$ and to perform clock division from the $20 \mathrm{~Gb} / \mathrm{s}$ stream to 10 and $5 \mathrm{GHz}$ [19]. The four subsystems allow the efficient solution of the following problems that are encountered in WDM and OTDM networks: (a) The generation of ultrashort pulses from a stable tunable source at $40 \mathrm{GHz}$ (fiber ring laser source) to provide the necessary optical power in the ultrafast WDM and OTDM networks, (b) The existence of simultaneous, synchronized wavelength channels $(10 \times 30 \mathrm{GHz}$ source). These may be used for clock recovery or synchronization purposes in the complex all optical circuits incorporating digital gates. They can also be deployed in networks combining WDM and OTDM as a single, cost effective source, (c) The synchronization of the all optical gates (AND, XOR) that are used in ultrafast OTDM networks with a single reference optical clock (clock repetition rate multiplier). These gates are key network elements because they perform all-optical functions such as demultiplexing (AND) and pattern matching (XOR) in the users access nodes, (d) The clock recovery (clock recovery/division circuit) operation that is essential for the generation of a high quality local clock synchronized with an incoming data stream to power the optical logic gates and to ensure data synchronization, despite the presence of jittered signal, in very high capacity WDM and OTDM networks.

A mathematical model of the fiber ring laser has been developed describing the mode - locking process in the laser oscillator and providing solutions for the steady - state mode - locked pulse profile. A complete investigation on the critical parameters that determine the width, energy and position of the mode - locked pulse relative to the external pulse has been performed. These parameters include the small signal gain and carrier lifetime of the SOA, the cavity loss and the pulse energy and width of the external modulating signal. From the analysis carried out it has been found that the minimum pulse width is obtained when these parameters are adjusted so that the mode - locked pulse forms in the middle between two 
successive external pulses and that this condition corresponds to the case where the energies of the external and mode - locked pulses are nearly equal. A rule of thumb has been also derived concerning the relationship between the operating repetition period and the carrier lifetime of the SOA which is particularly important when the fiber ring laser source is used for ultra - high speed all - optical applications.

\section{MODEL FOR MODE-LOCKING USING OPTICAL GAIN MODULATION OF A SEMICONDUCTOR OPTICAL AMPLIFIER}

Mode - locking is a well known technique for the generation of ultra short pulses and has been studied theoretically by several research groups [20 - 22]. In particular, active mode - locking has received considerable theoretical interest including analytical [23], [24] and numerical treatment [25], [26] for the estimation of mode - locked pulse characteristics.

This section presents the numerical model that describes the mode locking process of the SOA fiber ring cavity. The model follows the unidirectional propagation of the pulse around the cavity as it is shown in the block diagram of Figure 1. The operations considered inside the ring cavity are (a) saturable gain in the semiconductor laser optical amplifier (SOA) by the externally introduced pulse and the circulating mode-locked pulse, (b) bandwidth limitation of the circulating pulse with an optical filter and (c) linear losses that are present in the cavity.

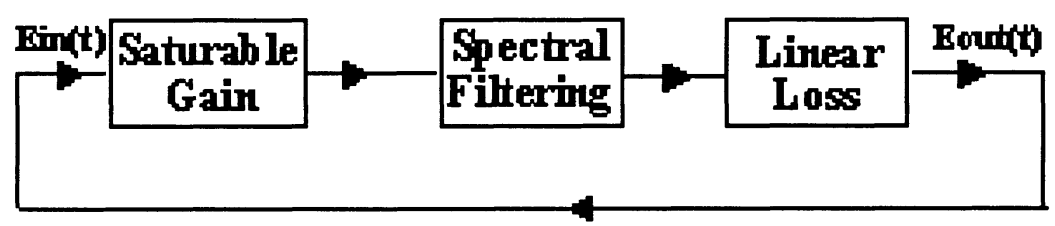

Figure 1. [Block diagram of fiber ring laser source used in simulation]

The parameters of the model are assumed to take values for which the oscillator operates under steady - state mode - locked condition. The pulse is therefore assumed to reproduce itself after one complete circulation around the laser cavity without any changes in the width or the energy, apart from a small temporal displacement. The other implicit assumptions which have been made in order to obtain the solutions are as follows: (a) The electric field of the circulating mode - locked pulse is assumed to be linearly polarized along the SOA axis of peak gain, (b) Changes in the mode - locked pulse profile due to its transit through each cavity element are small. As such, Taylor expansions of exponential terms to the first order incur small 
errors in the analysis, (c) The pulse energy of the external optical pulse and the circulating mode - locked pulse is assumed to be low enough compared to the saturation energy of the SOA. As a result of this, the gain coefficient of the SOA may be expanded up to second order with respect to pulse energies, (d) The bandwidth of the mode - locked pulse is assumed to be small compared to the bandwidth of the spectral limiting element in the cavity and Taylor expansion to second order of the pulse spectrum in the frequency domain is appropriate.

\subsection{Steady - state equations}

The profile of the output pulse after the consecutive transit through the SOA, spectral filtering and after encountering the cavity linear losses can be expressed as the successive operation of three operators $\hat{\mathrm{O}}_{1}, \hat{\mathrm{O}}_{2}$ and $\hat{\mathrm{O}}_{3}$ on the input pulse profile $E_{\text {in }}(t)$

$$
\begin{aligned}
\operatorname{Eout}(t)=\hat{O}_{1} \hat{O}_{2} \hat{O}_{3}[\operatorname{Ein}(t)] & =\exp \left[-\frac{L}{2}\right] \exp \left[\left(\frac{1}{2 \Delta \omega_{c}^{2}} \frac{d^{2}}{d t^{2}}\right)\right] \\
\cdot & \exp \left[\frac{G(t)}{2}\right] \operatorname{Ein}\left(t-T_{1}-T_{2}-T_{3}\right)
\end{aligned}
$$

where $T_{1}$ is the transit time of the pulse through the SOA, $T_{2}$ is the temporal delay that the pulse experiences when travelling from the exit of the SOA to the exit of the filter and $T_{3}$ is the temporal delay that the pulse experiences to travel from the exit of the filter to the entrance of the SOA. In this expression, $\hat{\mathrm{O}}_{1}$ is defined as the gain operator of the SOA acting on the electric field of the input pulses, $\hat{\mathrm{O}}_{1} \equiv \exp \left[\frac{\mathrm{G}(\mathrm{t})}{2}\right]$, with $\mathrm{G}(\mathrm{t})$ being the time - dependent power gain coefficient of the SOA, $\hat{\mathrm{O}}_{2}$ is defined as the time domain transfer operator of the pulse through the filter,

$$
\hat{\mathrm{O}}_{2} \equiv \exp \left[\frac{1}{2 \Delta \omega_{\mathrm{c}}^{2}} \frac{\mathrm{d}^{2}}{\mathrm{dt}^{2}}\right] \text {, where } \Delta \omega_{\mathrm{c}} \text { is related to the full width at half }
$$

maximum, $\Delta \omega_{\mathrm{f}}$, of $\Delta \omega_{\mathrm{c}}=\frac{1}{2 \sqrt{\ln 2}} \Delta \omega_{\mathrm{f}}$ the filter transfer function by and $\hat{\mathrm{O}}_{3}$ is defined as the linear loss operator, $\hat{\mathrm{O}}_{3} \equiv \exp \left[-\frac{\mathrm{L}}{2}\right]$, where $\mathrm{L}$ 
is the power linear loss coefficient of the system.

On the assumption that the profile change per pulse transit is small, the exponential terms in equation (1) may be expanded to first order providing

$$
E_{\text {out }}(t)=\left[1-\frac{L}{2}+\left(\frac{1}{2 \Delta \omega_{c}^{2}} \frac{d^{2}}{d t^{2}}+\frac{G(t)}{2}\right)\right] \operatorname{Ein}\left(t-T_{R}\right)
$$

where $T_{R}=T_{1}+T_{2}+T_{3}$ is the round trip time of the laser cavity, which for simplicity is assumed to be equal to the repetition period, $T_{\text {ext }}$, of the externally introduced pulse train.

In order to obtain closure and a steady - state solution for the mode locked pulse, the mode - locked pulse is assumed to reproduce itself after each complete transit through the SOA, filter and losses. The gain of the SOA is also assumed to recover to the same value before each mode - locked pulse enters it. In order to accommodate small temporal shifts, $\delta \mathrm{T}$, of the pulse around its peak as a result of the gain saturation in the SOA, the self reproducing pulse profile principle is applied by requiring that $E_{\text {out }}(t)=E_{i n}\left(t-T_{R}+\delta T\right)$ and by expanding to first order in $\delta T$ we obtain from (2)

$$
\left(1-g(t)-\frac{1}{L} \frac{1}{\Delta \omega_{c}^{2}} \frac{d^{2}}{{d t^{2}}^{2}}+\frac{\delta}{\Delta \omega_{c}} \frac{d}{d t}\right) \operatorname{Ein}(t)=0
$$

where we have introduced the normalized parameters $\delta=\frac{2}{\mathrm{~L}} \Delta \omega_{\mathrm{c}} \delta \mathrm{T}$ and

$$
g(t)=\frac{G(t)}{L}
$$

Specifically, $g(t)$ is the gain coefficient of the SOA normalized to the linear loss coefficient and $\delta$ is a time delay (or advance) parameter indicating whether the pulse repetition period deviates from the free space round trip time $T_{R}$.

The solution of this steady - state equation for the recirculating mode locked pulse requires the calculation of the time dependent normalized gain coefficient $\mathrm{g}(\mathrm{t})$. For this purpose, we consider in 2.2 the gain dynamics of the SOA during its gain saturation and recovery periods. In the analysis that follows, the external pulses are temporally separated from the mode - locked pulses by an unknown parameter, $\mathrm{T}_{\mathrm{s}}$, that is assumed to be large compared 
to the width of the two pulses. Physically, this means that the external pulse energy does not play any direct role in saturating the gain of the SOA during the presence of the mode - locked pulse and therefore need not be taken into account in equation (3) explicitly. The effect of the saturation of the SOA by the external pulses is however taken into account in calculating the input gain parameter at the entrance point of the mode - locked pulses as will be described in 2.4 .

\subsection{Semiconductor optical amplifier gain dynamics}

The ordinary differential equation that governs the temporal saturation for each point in the pulse is [27]

$$
\frac{d g(t)}{d t}=\frac{g_{s s}-g(t)}{t_{c a r}}-\frac{c n \varepsilon_{0} A}{2} \frac{\left|E_{i n}(t)\right|^{2}}{J_{\text {sat }}}\{\exp [g(t)]-1\}
$$

where $g_{s s}=\Gamma \sigma N_{o}\left(I / I_{0}-1\right) / L$ is the integrated small signal gain normalized to the cavity linear losses and $J_{\text {sat }}=\hbar \omega_{0} A / \sigma$ is the saturation energy of the amplifier. The parameters $t_{c a r}, \varepsilon_{0}, A, \Gamma, \sigma, \hbar \omega_{o}, N_{o}$ and $I_{o}$ are the carrier lifetime, the dielectric constant in vacuum, the cross section area of the active region, the confinement factor, the transition cross section, the photon energy and the carrier density and injection current required for transparency, respectively.

\subsection{Gain saturation by short optical pulse}

Equation (4) can be now used to obtain the gain saturation of the SOA for a short optical pulse, on the assumption that the width of the pulse is much smaller than the carrier lifetime of the amplifier [27]. This in practice means that the optical pulse is so short that the gain has no time to recover during its duration and the first term in the right hand side of equation (4) can be thus neglected. Assuming also for simplicity that around the peak of the mode - locked pulse $\mathrm{g}(\mathrm{t})$ is close to 1 so that the exponential term may be expanded to first order, the resulting normalized time dependent gain $\mathrm{g}(\mathrm{t})$ is

$$
g(t)=g_{\text {in }}^{p} \exp \left[-\frac{J_{\text {in }}(t)}{J_{\text {sat }}}\right]
$$


where $g_{\text {in }}^{p}$ is the gain before the arrival of the recirculating mode - locked

optical pulses and $\mathrm{J}_{\mathrm{in}}(\mathrm{t})=\frac{\mathrm{cn} \varepsilon_{\mathrm{o}} \mathrm{A}}{2} \int_{-\infty}^{\mathrm{t}}\left|\mathrm{E}_{\mathrm{in}}\left(\mathrm{t}^{\prime}\right)\right|^{2} \mathrm{dt}^{\prime} \quad$ is the instantaneous energy of the mode - locked pulses entering the SOA.

The exponential of (5) may be expanded, according to the assumptions already stated, into second order Taylor series and substituting in (3) yields

$$
\begin{aligned}
& {\left[1-g_{\text {in }}^{\mathrm{p}}+g_{\text {in }}^{\mathrm{p}} \frac{\mathrm{J}_{\text {in }}(\mathrm{t})}{\mathrm{J}_{\text {sat }}}-\frac{\mathrm{g}_{\text {in }}^{\mathrm{p}}}{2}\left(\frac{\mathrm{J}_{\text {in }}(\mathrm{t})}{\mathrm{J}_{\text {sat }}}\right)^{2}\right] \mathrm{E}_{\text {in }}(\mathrm{t})} \\
& +\frac{\delta}{\Delta \omega_{\mathrm{c}}} \frac{\mathrm{d} E_{\text {in }}(\mathrm{t})}{\mathrm{dt}}-\frac{1}{\mathrm{~L}} \frac{1}{\Delta \omega_{\mathrm{c}}^{2}} \frac{\mathrm{d}^{2} \operatorname{Ein}_{\text {in }}(\mathrm{t})}{\mathrm{dt}^{2}}=0
\end{aligned}
$$

The solution of this equation requires the knowledge of $g_{\text {in }}^{p}$ which can be found from the gain recovery analysis that follows.

\subsection{Gain recovery}

After the saturation of the SOA according to (5), the gain recovers due to the injection of carriers by the injection current. The gain recovery can be calculated, assuming that the stimulated recombination term (second term) in the right hand side of (4) can be neglected, from

$$
g(t)=\left(g_{f}-g_{s s}\right) \exp \left(-\frac{t}{t_{c a r}}\right)+g_{s s}
$$

where $g_{f}$ is the gain of the saturated SOA immediately after the transit of a pulse through given by

$$
g_{f}=g_{\text {in }} \exp \left[-\frac{J_{\text {total }}}{J_{\text {sat }}}\right]
$$


In this equation $g_{i n}$ and $J_{\text {total }}$ are the SOA gain and pulse energy immediately before the pulse entrance into the SOA. Given that two different sets of pulses transit the SOA at different times, i.e. the external and mode - locked pulses, the recovery of the SOA must be accounted separately for these sets of pulses. For this reason, $g_{\text {in }}$ takes the values $g_{\text {in }}^{\text {p }}$ and $g_{\text {in }}^{\text {ext }}$ and $J_{\text {total }}$ takes the values $J_{p}$ and $J_{\text {ext }}$ for the mode - locked and external pulses, respectively

In order to obtain the steady - state condition for the laser oscillator, the gain of the SOA is assumed to recover always to the same level $g_{\text {in }}^{p}$ and $g_{\text {in }}^{\text {ext }}$ before the mode - locked and the external pulses enter it, respectively. As the mode - locked pulse forms at time $T_{s}$ after the external pulse has entered the SOA, its gain has had $T_{s}-\Delta T$ time to recover to the value $g_{\text {in }}^{p}$ from the value $\mathrm{g}_{\mathrm{f}}^{\mathrm{ext}}$ before the arrival of the mode - locked pulse, where $\Delta \mathrm{T}=\frac{t_{\text {text }}+t_{p}}{2}$ with text being the width of the external pulse.

Similarly, the external pulse experiences a gain equal to $\mathrm{g}_{\text {in }}^{\text {ext }}$ which has recovered from the saturated value $g_{f}^{p}$ after the mode - locked pulse exits the SOA after $T_{R}-T_{s}-\Delta T$ time has elapsed. The equation of the recovery of the SOA is thus split in two equations which describe the SOA recovery after the transition of the external and mode - locked pulses, respectively. Equations (7) and (8) yield

$g_{\text {in }}^{p}=\left[g_{\text {in }}^{\text {ext }} \exp \left(-\frac{J_{\text {ext }}}{J_{\text {sat }}}\right)-g_{s s}\right] \exp \left(-\frac{T_{s}-\Delta T}{t_{c a r}}\right)+g_{s s}$

and

$g_{\text {in }}^{\text {ext }}=\left[g_{\text {in }}^{p} \exp \left(-\frac{J_{p}}{J_{s a t}}\right)-g_{s s}\right] \exp \left(-\frac{T_{R}-T_{s}-\Delta T}{t_{c a r}}\right)+g_{s s}$

Substituting (10) in (9), $\mathrm{g}_{\text {in }}^{\text {ext }}$ is eliminated and an expression is obtained which involves only $g_{\text {in }}^{\text {p }}$ 


$$
g_{\text {in }}^{p}=\frac{g_{s s}[1+\exp (A-\alpha)-\exp (A+B-\alpha)-\exp (A)]}{\left[1-\exp \left(A+B-U_{o}-\alpha\right)\right]}
$$

where $\quad U_{0}=\frac{J_{p}}{J_{\text {sat }}} \quad$ and $\quad \alpha=\frac{J_{\text {ext }}}{J_{\text {sat }}} \quad$ are the normalized to the SOA saturation energy energies of the mode - locked and external pulses

respectively, $\quad A=-\frac{T_{s}-\Delta T}{t_{c a r}}$ and $B=-\frac{T_{R}-T_{s}-\Delta T}{t_{c a r}}$.

\section{$2.5 \quad$ Solution}

In order to obtain a steady - state solution for the mode - locked pulse profile in terms of its width and energy, the mode - locked pulses are assumed to be squared hyperbolic secant in shape with total energy $J_{p}$ so that the electric field is given by

$$
\operatorname{Ein}(t)=\left(\frac{U_{o} J_{\text {sat }}}{2 t_{p}}\right)^{1 / 2} \operatorname{sech}\left(\frac{t}{t_{p}}\right)
$$

where $t_{p}$ is related to the full width at half maximum, $T_{F W H M}$, by

$$
\mathrm{t}_{\mathrm{p}}=\frac{\mathrm{T}_{\mathrm{FWHM}}}{\ln (3+2 \sqrt{2})} .
$$

Squaring (12) and integrating over the time dependent variable we obtain

$$
\frac{\mathrm{J}_{\text {in }}(\mathrm{t})}{\mathrm{J}_{\mathrm{sat}}}=\frac{\mathrm{U}_{\mathrm{o}}}{2}\left(1+\tanh \frac{\mathrm{t}}{\mathrm{t}_{\mathrm{p}}}\right)
$$

Substituting (12) and (13) and equating the coefficients of the same order terms in (6), the following equations are derived 


$$
\begin{aligned}
& 1-g_{\text {in }}^{p}+\frac{g_{\text {in }}^{p} U_{o}}{2}-\frac{g_{\text {in }}^{p} U_{o}^{2}}{8}-\frac{1}{L} \frac{1}{t_{p}^{2} \Delta \omega_{c}^{2}}=0 \\
& \frac{g_{\text {in }}^{p} U_{o}}{2}-\frac{g_{\text {in }}^{p} U_{o}^{2}}{4}-\frac{\delta}{t_{p} \Delta \omega_{c}}=0 \\
& \frac{2}{L_{p}^{2} \Delta \omega_{c}^{2}}-\frac{g_{\text {in }}^{p} U_{o}^{2}}{8}=0
\end{aligned}
$$

Equations (11), (14), (15) and (16) form a set of nonlinear equations with unknowns $t_{p}, U_{o}, g_{\text {in }}^{p}$, and $\delta$. Setting $\delta=\frac{x}{L_{p} \Delta \omega_{c}}$ we
obtain

$$
\begin{aligned}
& g_{\text {in }}^{p}=\frac{(x+4)^{2}}{(x+4)^{2}-4(x+1)} \\
& U_{o}=\frac{8}{x+4} \\
& t_{p} \Delta \omega_{c}=\frac{|x+4|}{2 \sqrt{g_{\text {in }}^{p} L}}
\end{aligned}
$$

Equations (11), (17), (18) and (19) form the final set of equations for the calculation of $t_{p} \Delta \omega_{c}$. 


\section{RESULTS OF THE SELF-CONSISTENT MODEL AND DISCUSSION}

\subsection{Calculation of $T_{\text {FWHM }} \Delta \omega_{\mathrm{f}}$}

The calculation of $t_{p} \Delta \omega_{c}$ and is performed by setting equation (11) equal to (17) and substituting the known values of the period and pulse width of the external pulse train, the SOA carrier lifetime and the cavity loss. The derived equation in $\mathrm{x}$ is however transcendental and requires numerical solution. In order to solve the transcendental equation and find the unknown $\mathrm{x}$, the parameters $\mathrm{a}$ and $\mathrm{T}_{\mathrm{s}}$ are scanned in accordance with the experimental conditions for a given value of the normalized small signal gain, $g_{s s}$, which is the only independent variable of the nonlinear system of equations. The unknown $\mathrm{x}$ is also scanned within a range whose lower limit is determined by the fact that the normalized energy of the mode - locked pulses must be always less than the normalized energy of the external pulses. Note that due to the form of the transcendental equation, the same combinations of the values of $U_{o}$ and $\alpha$ may correspond to multiple solutions of $x$. The selection of the single $\mathrm{x}$ solution and the elimination of the others is performed by choosing the value that corresponds to the smallest pulse width. Therefore, by solving the system for minimum pulse width, the optimum value of $\mathrm{T}_{\mathrm{s}}$ and $a$ is obtained for the given $g_{s s}$. The value of $x$ that corresponds to this set of $g_{s s}, \alpha$ and $T_{s}$ is then replaced in (19) to calculate $t_{p} \Delta \omega_{c}$ and the desired value of $T_{\text {FWHM }} \Delta \omega_{f}$ is finally obtained from

$$
\Delta \omega_{\mathrm{c}}=\frac{1}{2 \sqrt{\ln 2}} \Delta \omega_{\mathrm{f}} \quad \text { and } \quad \mathrm{t}_{\mathrm{p}}=\frac{\mathrm{T}_{\mathrm{FWHM}}}{\ln (3+2 \sqrt{2)}}
$$

\subsection{Simulation results and discussion}

Simulation results were obtained for repetition frequencies at $10 \mathrm{GHz}$ and 20 $\mathrm{GHz}$ in order to compare them to the experimental ones. The parameters used for the simulations were chosen to agree with the values in the experimental configuration [16]. Specifically, the width of the external pulses was $8 \mathrm{ps}$, the cavity loss was $15 \mathrm{~dB}$ and the full width at half maximum of the spectral limiting filter was $5 \mathrm{~nm}$ for both frequencies. The 
small signal power gain coefficient that was used in the calculation of the normalized to the loss small signal gain, $\mathrm{g}_{\mathrm{ss}}$ could take values in the range $19.2-25.2 \mathrm{~dB}$, in accordance to the gain characteristic of the SOA. The values also of the carrier lifetime were selected to be $100 \mathrm{ps}$ at $10 \mathrm{GHz}$ and $70 \mathrm{ps}$ at $20 \mathrm{GHz}$ in order to match the theoretical and experimental data provided for the $500 \mu \mathrm{m} \mathrm{SOA}$ that was used in the experiment. The decrease of the carrier lifetime at the higher repetition frequency is a result of the higher current at which the SOA has to be driven [28], [29] to account for twice the number of pulses entering the amplifier. The carrier lifetime is a particularly important parameter because it defines the range of repetition frequencies at which the ring laser may be operated for a given SOA, as it will be discussed with the simulation results at $20 \mathrm{GHz}$.

Figure 2 (a) shows the variation of the pulse width, $\mathrm{T}_{\mathrm{FWHM}}$, and the normalized energy, $\mathrm{U}_{\mathrm{o}}$, of the mode - locked pulse as well as of the required external pulse energy, $\alpha$, against the normalized small signal gain coefficient, $g_{s s}$, at $10 \mathrm{GHz}$. This figure shows that as the normalized small signal gain increases, the energy of the mode - locked pulse also increases saturating the SOA more strongly and leading to pulse narrowing. Similarly, the energy of the external pulse must also be increased to preserve the condition of shortest pulse from the oscillator. The width of the shortest mode - locked pulse decreases up to a minimum value as the normalized gain increases. The minimum pulse width was obtained for 1.53 normalized small signal gain and was $4.5 \mathrm{ps}$ assuming a filter bandwidth of $5 \mathrm{~nm}$ in the experimental system. The value of the mode - locked pulse energy, $U_{o}$, is either slightly less than or nearly equal to that of the external pulse, a. At the point of minimum pulse width, Uo takes the value 0.45 and nearly equals a which is 0.46 . The SOA is already heavily saturated at the same point and a further increase in the gain beyond this point requires a further increase in the energy of the external pulse, which does not allow an increase in the energy of the mode - locked pulse and results in an increase of its pulse width.

Figure 2 (b) illustrates the variation of the temporal separation, $\mathrm{T}_{\mathrm{s}}$, between the mode - locked and external pulses and for convenience the pulse width, $\mathrm{T}_{\mathrm{FWHM}}$, of the mode - locked pulses versus the normalized small signal gain, gss, at $10 \mathrm{GHz}$. This figure shows that provided the conditions for best pulse width are maintained, the mode - locked pulse forms at $48 \mathrm{ps,}$ approximately half way between two successive external pulses, despite large variations in gss. As the small signal gain increases, the mode - locked pulse moves away from the half way point and closer to the leading of the external pulses. This is due to the fact that as the small signal gain increases, the gain recovers above the losses in the cavity earlier, forcing the mode locked pulse to move forward. However, the excess gain increases very 
gradually so that the mode - locked pulse moves very slowly towards the leading of the two consecutive external pulses.

From the observation and analysis of Figures 2 (a) and (b) it can be deduced that two conditions must be fulfilled in order to obtain mode locked pulses with the minimum possible width. The first is that the normalized energies of the mode - locked and external pulses must be nearly equal. This condition alone is not sufficient since there is a large set of parameter values for which the normalized energies are equal but the pulse width is not globally minimum as seen in Figure 2 (b). This leads to the second condition which states that the mode - locked pulse must form nearly equidistantly between consecutive external pulses. When both conditions hold, the fiber ring laser system generates the shortest pulses. Figure 2 (c) displays the variation of the time delay, $\mathrm{T}_{\mathrm{s}}$, between the mode - locked and external pulses against the energy of the external pulse normalized to the saturation energy of the SOA. The results shown are for operation of the laser at $10 \mathrm{GHz}$ with the normalized small signal gain set at 1.53 . The figure shows that as the energy of the external pulse increases, the mode - locked pulse becomes increasingly delayed with respect to the leading of the two successive external pulses. This is a consequence of the deeper saturation into which the SOA is brought by the external pulse as its energy increases, which in turn requires a longer recovery time for the gain of the SOA to overcome the cavity losses and allow the mode - locked pulse to form. In the same figure the variation of the pulse width against the normalized external pulse energy has been plotted. As seen, the pulse width reaches a minimum value for $\alpha=0.46$ and forms roughly half way between two consecutive external pulses. Further increase of the external pulse energy beyond this point delays the formation of the mode - locked pulse further and results in its broadening.
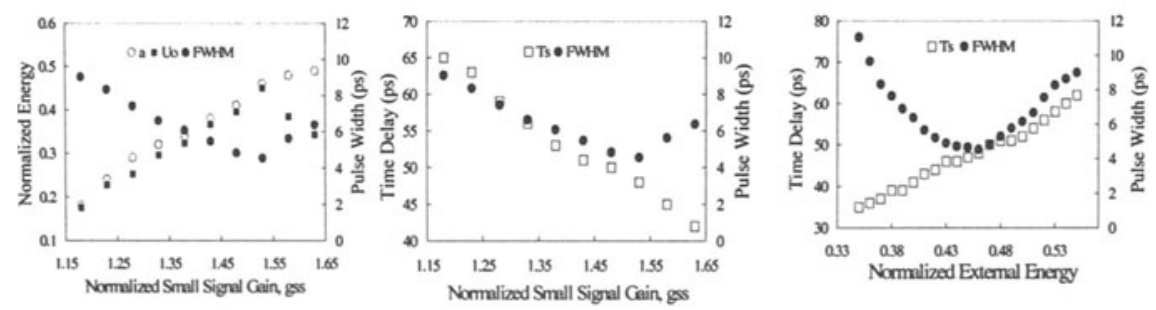

Figure 2. $10 \mathrm{GHz}$ simulation. (a) Variation of the mode - locked pulse width, FWHM, the normalized energy of the mode - locked , $\mathrm{U}_{0}$, and external pulses, $\mathrm{T}_{\mathrm{s}}$, versus the normalised small signal gain, $\mathrm{g}_{\mathrm{ss}}$. (b) Variation of the time separation between the mode - locked and external pulses, $T_{s}$, versus the normalized small signal gain, $g_{s s}$. (c) Variation of the time separation between the mode - locked and external pulses, $T_{s}$, versus the normalized energy of the external pulses, $\alpha$. 
The model was next used to evaluate the performance of the laser system as the repetition frequency of the external pulse train was increased to 20 $\mathrm{GHz}$. In order to examine the behaviour of the model, initially the same values for the physical parameters were used as in the case for $10 \mathrm{GHz}$ operation. This time, however, the width of the mode - locked pulses was longer than that of the external pulses. Note that an increase in the repetition rate of the oscillator restricts the range of values that $T_{s}$ may take, so that there is no overlap between the external and mode - locked pulses. The parameter that most critically determines the quality of mode - locking, however, is the carrier lifetime of the SOA. If the recovery time is far too short for a given repetition period, the gain of the SOA recovers too fast so that the mode - locked pulse forms early behind the external pulse. This allows excess gain to build at its trailing edge, resulting in longer mode locked pulses. On the contrary, if the recovery time is far too long, the mode - locked pulse trails too far behind the external pulse and has insufficient energy to strongly modulate the gain of the SOA, being therefore long again. In order to obtain a rule of thumb for the carrier lifetime, the theory of standard mode - locking is considered which stipulates that mode - locked pulses form in a laser cavity if the normalized gain function $g(t)$ is greater than 1 just as the pulse forms. In all other instances, that is ahead and behind the pulse, $g(t)$ must be smaller than 1 . In this sense and so as to ensure pulse formation, the carrier lifetime must be selected so that $g_{\text {in }}^{p}>1$ and $g_{f}^{p}<1$. Applying first and second order Taylor expansion approximations in (5) and (11), these inequalities hold simultaneously if

$$
1.1\left(\mathrm{~T}_{\mathrm{R}}-2 \mathrm{t}_{\mathrm{ext}}\right)<\mathrm{t}_{\mathrm{car}}<2.1\left(\mathrm{~T}_{\mathrm{R}}-2 \mathrm{t}_{\mathrm{ext}}\right)
$$

which sets a lower and an upper limit for the amplifier carrier lifetime with respect to the repetition period and the width of the external pulse. This condition implies that for the mode - locked pulses to form, the carrier lifetime of the SOA must decrease as the operating frequency of the ring laser increases. The inverse relationship between the carrier lifetime and the operating frequency may be explained if the roles of the frequency and small signal gain in the mode - locking process are considered. Specifically, an increase of the operating frequency requires an increase of the small signal gain in order to compensate the decrease in energy of the mode - locked pulses due to the larger number of pulses entering the amplifier per unit time. Experimentally, an increase of the small signal gain of the SOA is achieved with a higher drive current which also results in a decrease of its 
carrier lifetime [28], [29]. Following the preceding analysis, the carrier lifetime used in the $20 \mathrm{GHz}$ simulation was set to 70 ps.

Figure 3 (a) depicts the variation of the pulse width and the normalized energy of the mode - locked and external pulses as the normalized small signal gain coefficient is varied at $20 \mathrm{GHz}$. The behaviour of the oscillator is very similar to that presented in Figure 2 (a) for the $10 \mathrm{GHz}$ case. The minimum pulse width obtained was 3.9 ps for an increased normalized small signal gain of 1.83. Despite the increase in the small signal gain, the energies of the mode - locked and external pulses were lower than the $10 \mathrm{GHz}$ case, due to the heavy saturation of the SOA, but were again nearly equal at the point of minimum pulse width ( $U_{0}=0.36, a=0.37$ ). Note that if the same value of small signal gain as for the $10 \mathrm{GHz}$ operation is used, the mode - locked pulses are longer, $7 \mathrm{ps}$, with lower energy, $\mathrm{U}_{\mathrm{o}}=$ 0.22 .

Figure 3 (b) shows the variation of the time separation between the mode - locked and external pulses, $\mathrm{T}_{\mathrm{s}}$, versus gss. The pulse width of the mode locked pulses is also included so as to compare with the results from Figure 2 (b). The variation of the position of the mode - locked pulse at $20 \mathrm{GHz}$ has similar behaviour as at $10 \mathrm{GHz}$, that is, as the small signal gain increases, the mode - locked pulse moves forward towards the leading external pulse. The minimum pulse width is obtained for $\mathrm{T}_{\mathrm{s}}=25 \mathrm{ps}$, half way between two consecutive external pulses.
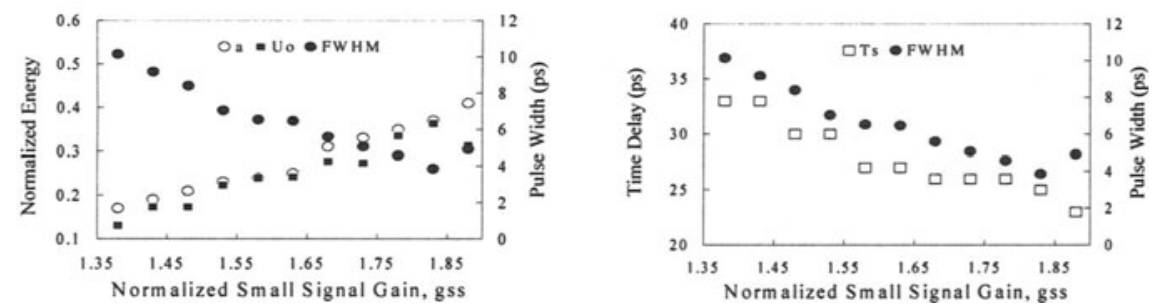

Figure 3. 20GHz simulation. (a) Variation of the mode - locked pulse width, FWHM, the normalized energy of the mode - locked, $U_{0}$, and external pulses, $\alpha$, versus the normalized small signal gain, $g_{s s}$. (b) Variation of the time separation between the mode - locked and external pulses, $T_{s}$, versus the normalized small signal gain, $g_{s s}$.

In order to assess the ability of the model for quantitative prediction, the parameter values from the experimental set up were substituted in equations (11), (14), (15) and (16). At $10 \mathrm{GHz}$, with an experimental value for gss 1.53 and using a filter of $5 \mathrm{~nm}$ spectral bandwidth and external modulating pulses of 8 ps width, the model predicts mode - locked pulses of 4.5 ps duration with normalized energy 0.45 and external pulses with normalized energy 0.46. In this instance the mode - locked pulse forms 48 ps behind the external pulse. At $20 \mathrm{GHz}$, for an increased normalized gain of 1.78, the 
model predicts mode - locked pulses of 4.5 ps duration and 0.34 normalized energy forming 26 ps behind the external pulses of 0.35 normalized energy. Experimentally, the shortest pulse train had 4.3 ps duration and formed 49 ps behind the external pulses at $10 \mathrm{GHz}$. At $20 \mathrm{GHz}$, the corresponding values were again 4.3 ps and 24 ps delay behind the external pulses. The agreement thus between the experimental and the theoretical values for the pulse width and the relative delay is exceptionally good given the simplicity of the model and suggests its robustness to the approximations that necessarily had to be made.

\section{CONCLUSION}

We have presented a theoretical study of the performance of a high repetition rate laser source that exploits the fast saturation of an intracavity semiconductor optical amplifier, forming the gain element in its cavity, from an external optical pulse to generate ultrashort pulses. A complete and computationally simple numerical model has been developed to provide solutions for the steady - state mode - locked pulse profile in terms of the critical parameters of the system. The results indicate that the minimum pulse width is obtained when these parameters are adjusted so that the mode - locked pulse forms in the middle between two successive external pulses and that this condition corresponds to the case where the energies of the external and mode - locked pulses are nearly equal. A practical rule of thumb has been also derived concerning the optimum combination of the SOA carrier lifetime and the repetition frequency that is required for the novel ring laser to be used as a high repetition rate source for transmission or optical logic experiments. The comparison of the experimental and theoretical results has revealed an excellent agreement between them and has helped to provide a thorough explanation of the mode - locking process.

\section{REFERENCES}

[1] S. Kawanishi, H. Takara, K. Uchiyama, I. Shake, K. Mori, Electron. Lett. 35 (1999) 826.

[2] K.L. Hall, K.A. Rauschenbach, Opt. Lett. 23 (1998) 1271.

[3] K. Zoiros, T. Houbavlis, K. Vlachos, H. Avramopoulos, F. Girardin, G. Guekos, S. Hansmann, H. Burkhard, CLEO Techn. Dig. CThF5 (1999) 379.

[4] J. D. Kafka, T. Bauer, D.W. Hall, Opt. Lett.14 (1989) 1269.

[5] D. Jones, H. Haus, E. Ippen, Opt. Lett. 21 (1996) 1818. 
[6] T. Pfeiffer, G. Veith, Electron. Lett. 29 (1993) 1849.

[7] E. Yoshida, Y. Kimura, M. Nakazawa, Electron. Lett. 31 (1995) 377.

[8] G.T. Harvey, L.F. Mollenauer, Opt. Lett. 18 (1993) 107.

[9] X. Shan, D.M. Spirit, Electron. Lett. 29 (1993) 979.

[10] M.J. Guy, J.R. Taylor, D.G. Moodie, A.E. Kelly, Electron. Lett. 32 (1996) 2240.

[11] T. Papakyriakopoulos, A. Stavdas, E.M. Protonotarios, H. Avramopoulos, Electron. Lett. 35 (1999) 717.

[12 ] S.W. Corzine, J.E. Bowers, G. Przybylek, U. Koren, B.I. Miller, C.E. Soccolich, Appl. Phys. Lett. 52 (1988) 348.

[13] R.S. Tucker, U. Koren, G. Raybon, C.A. Burrus, B.I. Miller, T.L. Koch, G. Eisenstein, Electron. Lett. 25 (1989) 621.

[14] H. Shi, G.A. Alphonse, J.C. Conolly, P.J. Delfyett, Electron. Lett. 34 (1998) 179.

[15] C. Schimdt, E. Dietrich, S. Diez, H.J. Ehrke, U. Feiste, L. Kuller, R. Ludwig, H.G. Weber, CLEO Techn. Dig. CThA3 (1999) 348.

[16] T. Papakyriakopoulos, K. Vlachos, A. Hatziefremidis, H. Avramopoulos, Opt. Lett. 24 (1999) 1209.

[17] K. Vlachos, K. Zoiros, T. Houbavlis, H. Avramopoulos, to appear in IEEE Photon. Technol. Lett., Jan. 2000

[18] T. Papakyriakopoulos, K. Vlachos, A. Hatziefremidis, H. Avramopoulos, Opt. Lett. 24 (1999) 717.

[19] K. Vlachos, T. Papakyriakopoulos, T. Houbavlis, H. Avramopoulos, ECOC Proc. (1999) I - 414.

[20] D.J. Kuizenga, A.E. Siegman, IEEE J. Quantum Electron. 6 (1970) 694.

[21] G.H.C. New, IEEE J. Quantum Electron. 10 (1974) 115.

[22] H.A. Haus, Japan. J. Appl. Phys. 20 (1981) 1007.

[23] H.A. Haus, J. Appl. Phys. 51 (1980) 4042.

[24] M. Schell, A.G. Weber, E.H. Boettcher, E. Scholl, D. Bimberg, IEEE J. Quantum Electron. 27 (1991) 402.

[25] M. Schell. A.G. Weber, E. Scholl, D. Bimberg, IEEE J. Quantum Electron. 27 (1991) 1661.

[26] P.J. Delfyett, A. Dienes, J.P. Heritage, M.Y. Hong, Y.H. Chang, Appl. Phys. B 58 (1994) 183.

[27] G. Agrawal and N.A. Olsson, IEEE J. Quantum Electron. 25 (1989) 2297.

[28] F. Girardin, G. Guekos, A. Houbavlis, IEEE Photon. Technol. Lett. 10 (1998) 784.

[29] G. Eisenstein, R.S. Tucker, J.M. Wiesenfeld, P.B. Hansen, G. Raybon, B.C. Johnson, T.J. Bridges, F.G. Storz, C.A Burrus, Appl. Phys. Lett. 54 (1989) 454. 